\title{
Concomitant Proton Pump Inhibitors and Immune Checkpoint Inhibitors Increase Nephritis Frequency
}

\author{
KOKI KATO $^{1 *}$, TOMOHIRO MIZUNO ${ }^{1 *}$, TAKENAO KOSEKI ${ }^{1 *}$, YOSHIMASA ITO $^{2}$, \\ MASAKAZU HATANO ${ }^{1}$, KAZUO TAKAHASHI ${ }^{3}$, SHIGEKI YAMADA ${ }^{1}$ and NAOTAKE TSUBOI ${ }^{2}$ \\ ${ }^{1}$ Department of Clinical Pharmacy, Fujita Health University School of Medicine, Toyoake, Japan; \\ ${ }^{2}$ Department of Nephrology, Fujita Health University School of Medicine, Toyoake, Japan; \\ ${ }^{3}$ Department of Biomedical Molecular Sciences, Fujita Health University School of Medicine, Toyoake, Japan
}

\begin{abstract}
Background/Aim: Concomitant proton pump inhibitor (PPI) and immune checkpoint inhibitor (ICPI) were determined as risk factors of acute kidney injury. To identify the type of PPI associated with ICPI-induced nephritis, we used the Japanese Adverse Drug Event Report database. Patients and Methods: ICPIs (nivolumab, pembrolizumab, ipilimumab, atezolizumab, durvalumab, and avelumab) and PPIs (esomeprazole, omeprazole, vonoprazan, rabeprazole, and lansoprazole) were selected as suspected nephritis-inducing drugs. Results: The cases of concomitant use of atezolizumab and rabeprazole, ipilimumab and omeprazole, ipilimumab and lansoprazole, nivolumab and esomeprazole, nivolumab and omeprazole, nivolumab and rabeprazole, nivolumab and lansoprazole, pembrolizumab and esomeprazole, as well as pembrolizumab and lansoprazole had a significantly higher reported odds ratio than monotherapy cases. Conclusion: Male patients or patients using ICPIs and PPIs (excluded vonoprazan) concomitantly should be monitored for renal function after chemotherapy.
\end{abstract}

Immune checkpoint inhibitors (ICPIs) are used as essential anti-cancer chemotherapy in various types of cancers (1-5). Blockade of programmed cell death-1 (PD-1)/PD-ligand-1 signaling $(6,7)$ and cytotoxic T-lymphocyte-associated protein 4 (CTLA-4) signaling (8) activates T-cell mediated

This article is freely accessible online.

*These Authors contributed equally to this study.

Correspondence to: Tomohiro Mizuno, Ph.D., Department of Clinical Pharmacy, Fujita Health University School of Medicine, 1-98 Dengakugakubo, Kutsukake-cho, Toyoake, 470-1192, Japan. Tel: +81 562932493, Fax: +81 562934593, e-mail: tomohiro.mizuno@fujita-hu.ac.jp

Key Words: Proton pump inhibitor, immune checkpoint inhibitor, nephritis, Japanese adverse drug event report. antitumor immunity; therefore, ICPIs exert dramatic effects in patients with cancer expressing these proteins. As ICPIs induce antitumor effects by reactivating antitumor immunity, they also cause immune-related adverse events (irAEs), such as interstitial pneumonia and nephritis (9-11), thyroid dysfunction $(5,9)$, type 1 diabetes mellitus (5), and lupus erythematosus (12). The clinical features and outcomes of ICPI-induced acute kidney injury (AKI) have been reported (13-15). As a pathological feature, acute tubulointerstitial nephritis was the primary pathologic lesion with lymphocyte infiltration. In addition, lower baseline estimated glomerular filtration rate (eGFR), use of proton pump inhibitor (PPI), and ICPI combination were determined as risk factors of ICPI-associated AKI (13). Furthermore, the mortality of patients with renal recovery after ICPI-induced nephritis was better than that of patients without renal recovery (16). To improve prognosis for patients treated with ICPIs, the prevention of ICPI-induced nephritis is essential.

PPIs are traditionally widely used for the treatment of several acid-related disorders, including peptic ulcer disease, gastroesophageal reflux disease, and Helicobacter pylori eradication. Although the use of PPIs was perceived as safe, it is associated with the incidence of AKI (17-22). In particular, omeprazole is associated with acute interstitial nephritis (AIN) (17). Because AKI and AIN increase the risk of chronic kidney disease (CKD), the prevention of PPIinduced AIN could decrease the initiation of dialysis (23-25).

Since the frequency of overall incidence of ICPI-induced AKI is $2.2 \%$ (26), the information regarding ICPI-induced AKI is limited. In addition, it remains unclear as to which PPIs increase the risk of AKI. The Japanese Adverse Drug Event Report (JADER) database is an open-access database of adverse drug events (ADEs). The JADER database is useful for calculating ADE signals in rare cases. The frequencies of irAEs associated with ICPIs were approximately 50\% (skin disorders), $40 \%$ (gastrointestinal disorders), $8 \%$ (endocrine disorders), $4 \%$ (hepatitis), and $1 \%$ (pneumonitis) in advanced melanoma (14). Because of 
their low frequency, the signals of irAEs for nephritis/renal dysfunction, pneumonitis, rash, and type 1 diabetes mellitus associated with ICPIs were calculated using JADER (27). However, information on drug-drug interactions is limited. In this study, we aimed to elucidate the type of PPI associated with ICPI-induced nephritis and used the JADER database.

\section{Patients and Methods}

Data source. Data from April 2004 to September 2020 were extracted from the JADER database. The JADER database consists of four data tables: patient demographic information (demo), drug information (drug), ADEs (reac), and primary disease (hist). The duplicated data in the "drug" and "reac" tables were removed, and the "demo" table was linked to the "drug" and "reac" tables using each case identified in the data tables. In these cases, the contribution of the medications to the ADEs was classified into three categories: "suspected medicine", "concomitant medicine", and "interaction". The "suspected medicine" category was extracted into ADEs in the present study.

The "demo" table contained data for patient sex and age, as well as other patient characteristics. Data without sex or age information were excluded from the dataset. For the association analysis performed with patients classified in 10-year age intervals, we defined "older adults" as those in their "70s", "80s", "90s", and "100s", according to a previous report (28). Nivolumab, pembrolizumab, ipilimumab, atezolizumab, durvalumab, and avelumab were selected as suspected drugs for analysis of irAEs. These ICPIs have been approved by the Japanese Ministry of Health, Labor, and Welfare.

Definition of cancer patients. The primary disease in the "hist" tables was defined on the basis of the preferred terms (PTs) in the Medical Dictionary for Regulatory Activities (MedDRA) version 23.1. MedDRA term grouping at the PT level defines the patient's medical condition. Cancer as a primary disease as defined by PTs is shown in Table I after removing duplicated data. Other cancers not included in Table I that appeared as primary diseases were classified as others/uncertain.

Definition of ICPIs and nephritis as irAEs. ICPIs (nivolumab, pembrolizumab, ipilimumab, atezolizumab, durvalumab, and avelumab) and PPIs (esomeprazole, omeprazole, vonoprazan, rabeprazole, and lansoprazole) were selected as suspected nephritisinducing drugs. The ADEs in the "reac" table were coded according to the PTs in the MedDRA. Nephritis as an irAE was selected by three nephrologists from the MedDRA, and the PTs for nephritis are listed in Table II.

Statistical analysis. The reporting odds ratio (ROR), which serves as an index for adverse event signals, was calculated using the following equations (28), with a, b, c, and d cross-tabulation as follows: a, number of cases with an ADE related to the use of the suspected drug; b, number of cases with an ADE related to the use of all other drugs; c, number of cases with all other ADEs related to the use of the suspected drug; and d, number of cases with all other ADEs related to the use of all other drugs.

$\mathrm{ROR}=(\mathrm{a} / \mathrm{b}) /(\mathrm{c} / \mathrm{d})=\mathrm{ad} / \mathrm{bc}$
Adverse event signals were recognized as significant when the ROR estimates and the lower limits of the corresponding $95 \%$ confidence interval (CI) exceeded 1 . RORs were calculated using Excel for Microsoft 365 (Microsoft Corporation, Redmond, WA, USA). The signals of drug-drug interactions were evaluated as significant when the lower limits of the corresponding 95\% CI in drug-drug interactions exceeded the higher limits of the corresponding 95\% CI in monotherapy (29).

Chi-square test as univariate analysis and multiple logistic regression analysis were used to assess the risk of nephritis in ICPI monotherapy. Two-sided $p$-values less than 0.05 were considered significant. We conducted the multiple logistic regression analysis in ICPI dataset showing significant ADE signals of nephritis. Multiple logistic regression analysis in each ICPI dataset was performed using SPSS version 22.0 (SPSS Inc., Chicago, IL, USA).

\section{Results}

Patient characteristics and ROR of monotherapy. A total of 591,114 cases were included in the dataset (Figure 1). The numbers of side-effects associated with nivolumab, pembrolizumab, ipilimumab, atezolizumab, durvalumab, and avelumab were 9,116, 5,838, 2,831, 1,288, 1,054, and 48, respectively (Table III). In patients taking ICPI monotherapy, ADE signals of nephritis were detected in the atezolizumab, ipilimumab, durvalumab, nivolumab, and pembrolizumab groups. The use of atezolizumab [ROR $(95 \% \mathrm{CI})=1.780$ (1.102 to 2.874$)$ ], ipilimumab [ROR $(95 \% \mathrm{CI})=2.454(1.857$ to 3.242$)$ ], nivolumab [ROR $(95 \% \mathrm{CI})=2.091(1.764$ to $2.479)]$, and pembrolizumab $[\mathrm{ROR}(95 \% \mathrm{CI})=2.443$ (2.008 to 2.973)] showed a statistically significant signal for nephritis (Table IV). Although the durvalumab group also showed ADE signals for nephritis, the signals were not statistically significant [ROR $(95 \% \mathrm{CI})=0.252(0.063$ to 1.010$)]$ (Table IV). Moreover, the avelumab group did not show any ADE signal for nephritis because of the small sample size.

ADE signals of nephritis were detected in patients treated with PPIs. The use of esomeprazole [ROR $(95 \% \mathrm{CI})=2.064$ (1.326 to 3.214)], omeprazole [ROR $(95 \% \mathrm{CI})=4.248$ (3.209 to 5.622)], vonoprazan [ROR $(95 \% \mathrm{CI})=1.829(1.132$ to 2.954)], rabeprazole [ROR $(95 \% \mathrm{CI})=3.169$ (2.263 to 4.437$)$ ], and lansoprazole [ROR $(95 \% \mathrm{CI})=2.178(1.705$ to 2.783$)]$ showed a statistically significant signal for nephritis (Table IV).

Drug-drug interaction signals. The signals of drug-drug interactions are shown in Table IV. Cases with concomitant use of atezolizumab and rabeprazole [ROR $(95 \% \mathrm{CI})=66.43(6.022$ to 732.8)], ipilimumab and omeprazole $[\mathrm{ROR}(95 \% \mathrm{CI})=265.8$ (24.09 to 2931)], ipilimumab and lansoprazole [ROR (95\% $\mathrm{CI})=29.53(6.378$ to 136.7$)]$, nivolumab and esomeprazole [ROR $(95 \% \mathrm{CI})=40.91(13.33$ to 125.5$)]$, nivolumab and omeprazole [ROR (95\% CI $)=199.5 \quad(56.28$ to 707.3$)$ ], nivolumab and rabeprazole $[\mathrm{ROR}(95 \% \mathrm{CI})=114.0$ (38.30 to 339.4)], nivolumab and lansoprazole $[\mathrm{ROR}(95 \% \mathrm{CI})=31.04$ (13.67 to 70.71)], pembrolizumab and esomeprazole [ROR 
Kato et al: Concomitant PPIs and ICPIs Increase Nephritis Frequency

Table I. Preferred terms to define different cancer types (appearing in the Medical Dictionary for Regulatory Activities version 23.1).

\begin{tabular}{|c|c|c|c|c|c|}
\hline $\begin{array}{l}\text { Cancer } \\
\text { type }\end{array}$ & $\begin{array}{l}\text { Preferred terms } \\
\text { number }\end{array}$ & Preferred terms & $\begin{array}{l}\text { Cancer } \\
\text { type }\end{array}$ & $\begin{array}{c}\text { Preferred terms } \\
\text { number }\end{array}$ & Preferred terms \\
\hline \multirow{40}{*}{$\begin{array}{l}\text { Non-small } \\
\text { cell lung } \\
\text { cancer }\end{array}$} & \multirow[t]{3}{*}{10001245} & \multirow[t]{3}{*}{ Adenosquamous cell lung cancer } & & 10005003 & Bladder cancer \\
\hline & & & & 10005005 & Bladder cancer recurrent \\
\hline & & & & 10005006 & Bladder cancer stage 0 , with cancer in situ \\
\hline & 10001247 & Adenosquamous cell lung cancer recurrent & & 10005008 & Bladder cancer stage I, with cancer in situ \\
\hline & 10001248 & Adenosquamous cell lung cancer stage 0 & & 10005010 & Bladder cancer stage II \\
\hline & 10001249 & Adenosquamous cell lung cancer stage I & & 10005011 & Bladder cancer stage III \\
\hline & 10001250 & Adenosquamous cell lung cancer stage II & & 10005012 & Bladder cancer stage IV \\
\hline & 10001251 & Adenosquamous cell lung cancer stage III & & $10005075 \mathrm{I}$ & Bladder squamous cell carcinoma recurrent \\
\hline & 10001254 & Adenosquamous cell lung cancer stage IV & & 10005076 & Bladder squamous cell carcinoma stage 0 \\
\hline & 10023775 & Large cell lung cancer recurrent & & 10005077 & Bladder squamous cell carcinoma stage I \\
\hline & 10023776 & Large cell lung cancer stage 0 & & 10005078 & Bladder squamous cell carcinoma stage II \\
\hline & 10023777 & Large cell lung cancer stage I & & 10005079 & Bladder squamous cell carcinoma stage III \\
\hline & 10023778 & Large cell lung cancer stage II & & 10005080 & Bladder squamous cell carcinoma stage IV \\
\hline & 10023779 & Large cell lung cancer stage III & & 10005081 & Bladder squamous cell carcinoma stage \\
\hline & 10023780 & Large cell lung cancer stage IV & & & unspecified \\
\hline & 10025031 & Lung adenocarcinoma & & 10005084 & Bladder transitional cell carcinoma \\
\hline & 10025033 & Lung adenocarcinoma recurrent & & 10057352 & Metastatic carcinoma of the bladder \\
\hline & 10025034 & Lung adenocarcinoma stage 0 & & 10066749 & Bladder transitional cell carcinoma stage 0 \\
\hline & 10025035 & Lung adenocarcinoma stage I & & 10066750 & Bladder transitional cell carcinoma \\
\hline & 10025036 & Lung adenocarcinoma stage II & & & recurrent \\
\hline & 10025037 & Lung adenocarcinoma stage III & & 10066751 & Bladder transitional cell carcinoma stage I \\
\hline & 10025038 & Lung adenocarcinoma stage IV & & $10066752 \mathrm{~F}$ & Bladder transitional cell carcinoma stage IV \\
\hline & 10025120 & Lung squamous cell carcinoma recurrent & & 10066753 & Bladder transitional cell carcinoma stage II \\
\hline & 10025121 & Lung squamous cell carcinoma stage 0 & & 10066754 & Bladder transitional cell carcinoma \\
\hline & 10025122 & Lung squamous cell carcinoma stage I & & & stage III \\
\hline & 10025123 & Lung squamous cell carcinoma stage II & & 10071664 & Bladder transitional cell carcinoma \\
\hline & 10025124 & Lung squamous cell carcinoma stage III & & & metastatic \\
\hline & 10025125 & Lung squamous cell carcinoma stage IV & & 10078341 & Neuroendocrine carcinoma of the bladder \\
\hline & 10029515 & Non-small cell lung cancer recurrent & & 10026426 & Malignant neoplasm of renal pelvis \\
\hline & 10029516 & Non-small cell lung cancer stage 0 & & 10044406 & Transitional cell cancer of renal pelvis and \\
\hline & 10029517 & Non-small cell lung cancer stage I & & & ureter metastatic \\
\hline & 10029518 & Non-small cell lung cancer stage II & & 10044407 & Transitional cell cancer of the renal pelvis \\
\hline & 10029519 & Non-small cell lung cancer stage III & & & and ureter \\
\hline & 10029520 & Non-small cell lung cancer stage IIIA & & 10044408 & Transitional cell cancer of the renal pelvis \\
\hline & 10029521 & Non-small cell lung cancer stage IIIB & & & and ureter localised \\
\hline & 10029522 & Non-small cell lung cancer stage IV & & 10044410 & Transitional cell cancer of the renal pelvis \\
\hline & 10061873 & Non-small cell lung cancer & & & and ureter recurrent \\
\hline & 10069730 & Large cell lung cancer metastatic & & 10044411 & Transitional cell cancer of the renal pelvis \\
\hline & 10071533 & Lung squamous cell carcinoma metastatic & & & and ureter regional \\
\hline & 10059515 & Non-small cell lung cancer metastatic & & 10046392 & Ureteric cancer \\
\hline Head and & 10071540 & Head and neck cancer metastatic & & 10046393 & Ureteric cancer local \\
\hline neck cancer & & & & 10046394 & Ureteric cancer metastatic \\
\hline & 10067821 & Head and neck cancer & & 10046396 & Ureteric cancer recurrent \\
\hline & 10071539 & Head and neck cancer stage I & & 10046397 & Ureteric cancer regional \\
\hline & 10071538 & Head and neck cancer stage II & & 10026326 & Malignant neoplasm of paraurethral glands \\
\hline & 10071537 & Head and neck cancer stage III & & 10044412 & Transitional cell carcinoma \\
\hline & 10071536 & Head and neck cancer stage IV & & 10044426 & Transitional cell carcinoma urethra \\
\hline & 10060121 & Squamous cell carcinoma of head and neck & & 10046431 & Urethral cancer \\
\hline Urothelial & 10004986 & Bladder adenocarcinoma recurrent & & 10046433 & Urethral cancer metastatic \\
\hline & & & & 10046435 & Urethral cancer recurrent \\
\hline & 10004987 & Bladder adenocarcinoma stage 0 & & 10061272 & Malignant urinary tract neoplasm \\
\hline & 10004988 & Bladder adenocarcinoma stage I & & 10061396 & Urinary tract carcinoma in situ \\
\hline & 10004989 & Bladder adenocarcinoma stage II & & 10071080 & Transitional cell carcinoma metastatic \\
\hline & 10004990 & Bladder adenocarcinoma stage III & & 10074419 & Malignant genitourinary tract neoplasm \\
\hline & 10004991 & Bladder adenocarcinoma stage IV & & 10077051 & Transitional cell carcinoma recurrent \\
\hline & 10004992 & Bladder adenocarcinoma stage unspecified & & 10005056 & Bladder neoplasm \\
\hline
\end{tabular}

Table I. Continued 
in vivo $35: 2831-2840(2021)$

Table I. Continued.

\begin{tabular}{|c|c|c|c|c|c|}
\hline $\begin{array}{l}\text { Cancer } \\
\text { type }\end{array}$ & $\begin{array}{c}\text { Preferred terms } \\
\text { number }\end{array}$ & Preferred terms & $\begin{array}{l}\text { Cancer } \\
\text { type }\end{array}$ & $\begin{array}{l}\text { Preferred terms } \\
\text { number }\end{array}$ & Preferred terms \\
\hline \multirow{34}{*}{$\begin{array}{l}\text { Renal cell } \\
\text { carcinoma }\end{array}$} & 10061398 & Urinary tract neoplasm & & 10020209 & Hodgkin's disease lymphocyte depletion \\
\hline & 10062221 & Ureteral neoplasm & & & stage I subdiaphragm \\
\hline & 10062223 & Urethral neoplasm & & 10020210 & Hodgkin's disease lymphocyte depletion \\
\hline & 10009253 & Clear cell sarcoma of the kidney & & & stage I supradiaphragm \\
\hline & & & & 10020211 & Hodgkin's disease lymphocyte depletion \\
\hline & 10029145 & Nephroblastoma & & & stage II site unspecified \\
\hline & 10038389 & Renal cancer & & 10020212 & Hodgkin's disease lymphocyte depletion \\
\hline & 10038390 & Renal cancer recurrent & & & stage II subdiaphragm \\
\hline & 10038391 & Renal cancer stage I & & 10020213 & Hodgkin's disease lymphocyte depletion \\
\hline & 10038392 & Renal cancer stage II & & & stage II supradiaphragm \\
\hline & 10038393 & Renal cancer stage III & & 10020215 & Hodgkin's disease lymphocyte depletion \\
\hline & 10038394 & Renal cancer stage IV & & & type recurrent \\
\hline & 10038410 & Renal cell carcinoma recurrent & & 10020216 & Hodgkin's disease lymphocyte depletion \\
\hline & 10038411 & Renal cell carcinoma stage I & & & type refractory \\
\hline & 10038412 & Renal cell carcinoma stage II & & 10020217 & Hodgkin's disease lymphocyte depletion \\
\hline & 10038413 & Renal cell carcinoma stage III & & & type stage III \\
\hline & 10038414 & Renal cell carcinoma stage IV & & 10020218 & Hodgkin's disease lymphocyte depletion \\
\hline & 10039019 & Rhabdoid tumour of the kidney & & & type stage IV \\
\hline & 10050018 & Renal cancer metastatic & & 10020219 & Hodgkin's disease lymphocyte depletion \\
\hline & 10050176 & Renal oncocytoma & & & type stage unspecified \\
\hline & 10050513 & Metastatic renal cell carcinoma & & 10020220 & Hodgkin's disease lymphocyte \\
\hline & 10051948 & Renal adenoma & & & predominance stage I site unspec \\
\hline & 10061482 & Renal neoplasm & & 10020221 & Hodgkin's disease lymphocyte \\
\hline & 10061872 & Non-renal cell carcinoma of kidney & & & predominance stage I subdiaphragm \\
\hline & 10067943 & Hereditary papillary renal carcinoma & & 10020222 & Hodgkin's disease lymphocyte \\
\hline & 10067944 & Hereditary leiomyomatosis renal & & & predominance stage I supradiaphragm \\
\hline & & cell carcinoma & & 10020223 & Hodgkin's disease lymphocyte \\
\hline & 10067946 & Renal cell carcinoma & & & predominance stage II site unspec \\
\hline & 10069908 & Renal haemangioma & & 10020224 & Hodgkin's disease lymphocyte \\
\hline & 10073251 & Clear cell renal cell carcinoma & & & predominance stage II subdiaphragm \\
\hline & 10078493 & Papillary renal cell carcinoma & & 10020225 & Hodgkin's disease lymphocyte \\
\hline & 10080544 & Chromophobe renal cell carcinoma & & & predominance stage II supradiaphragm \\
\hline & 10081895 & Multilocular cystic nephroma & & 10020227 & Hodgkin's disease lymphocyte \\
\hline & 10083207 & Renal hamartoma & & & predominance type recurrent \\
\hline \multirow[t]{7}{*}{ Melanoma } & 10025650 & Malignant melanoma & & 10020228 & Hodgkin's disease lymphocyte \\
\hline & 10025652 & Malignant melanoma in situ & & & predominance type refractory \\
\hline & 10025668 & Malignant melanoma stage I & & 10020229 & Hodgkin's disease lymphocyte \\
\hline & 10025669 & Malignant melanoma stage II & & & predominance type stage III \\
\hline & 10025670 & Malignant melanoma stage III & & 10020230 & Hodgkin's disease lymphocyte \\
\hline & 10025671 & Malignant melanoma stage IV & & & predominance type stage IV \\
\hline & 10027480 & Metastatic malignant melanoma & & 10020231 & Hodgkin's disease lymphocyte \\
\hline \multirow{13}{*}{$\begin{array}{l}\text { Gastric } \\
\text { cancer }\end{array}$} & 10001150 & Adenocarcinoma gastric & & & predominance type stage unspecified \\
\hline & 10017758 & Gastric cancer & & 10020206 & Hodgkin's disease \\
\hline & 10017761 & Gastric cancer recurrent & & 10020266 & Hodgkin's disease recurrent \\
\hline & 10017762 & Gastric cancer stage 0 & & 10020267 & Hodgkin's disease refractory \\
\hline & 10017763 & Gastric cancer stage I & & 10020268 & Hodgkin's disease stage I \\
\hline & 10017764 & Gastric cancer stage II & & 10020269 & Hodgkin's disease stage II \\
\hline & 10017765 & Gastric cancer stage III & & 10020270 & Hodgkin's disease stage III \\
\hline & 10055008 & Gastric sarcoma & & 10020271 & Hodgkin's disease unclassifiable \\
\hline & 10061967 & Gastric cancer stage IV & & 10061597 & Hodgkin's disease stage IV \\
\hline & 10062878 & Gastrooesophageal cancer & & 10020233 & Hodgkin's disease mixed cellularity \\
\hline & 10063916 & Metastatic gastric cancer & & & recurrent \\
\hline & 10066896 & HER2 positive gastric cancer & & 10020234 & Hodgkin's disease mixed cellularity \\
\hline & 10081398 & Gastrooesophageal cancer recurrent & & & refractory \\
\hline $\begin{array}{l}\text { Hodgkin } \\
\text { lymphoma }\end{array}$ & 10020208 & $\begin{array}{l}\text { Hodgkin's disease lymphocyte depletion } \\
\text { stage I site unspecified }\end{array}$ & & 10020235 & $\begin{array}{l}\text { Hodgkin's disease mixed cellularity } \\
\text { stage I site unspecified }\end{array}$ \\
\hline
\end{tabular}


Table I. Continued.

\begin{tabular}{|c|c|c|}
\hline $\begin{array}{l}\text { Cancer } \\
\text { type }\end{array}$ & $\begin{array}{c}\text { Preferred terms } \\
\text { number }\end{array}$ & Preferred terms \\
\hline & 10020236 & $\begin{array}{l}\text { Hodgkin's disease mixed cellularity } \\
\text { stage I subdiaphragmatic }\end{array}$ \\
\hline & 10020237 & $\begin{array}{l}\text { Hodgkin's disease mixed cellularity } \\
\text { stage I supradiaphragmatic }\end{array}$ \\
\hline & 10020238 & $\begin{array}{l}\text { Hodgkin's disease mixed cellularity } \\
\text { stage II subdiaphragmatic }\end{array}$ \\
\hline & 10020239 & $\begin{array}{l}\text { Hodgkin's disease mixed cellularity } \\
\text { stage II supradiaphragmatic }\end{array}$ \\
\hline & 10020240 & $\begin{array}{c}\text { Hodgkin's disease mixed cellularity } \\
\text { stage III }\end{array}$ \\
\hline & 10020241 & $\begin{array}{c}\text { Hodgkin's disease mixed cellularity } \\
\text { stage IV }\end{array}$ \\
\hline & 10020242 & $\begin{array}{l}\text { Hodgkin's disease mixed cellularity } \\
\text { stage unspecified }\end{array}$ \\
\hline & 10020244 & Hodgkin's disease nodular sclerosis \\
\hline & 10020245 & $\begin{array}{l}\text { Hodgkin's disease nodular } \\
\text { sclerosis recurrent }\end{array}$ \\
\hline & 10020246 & $\begin{array}{l}\text { Hodgkin's disease nodular } \\
\text { sclerosis refractory }\end{array}$ \\
\hline & 10020252 & $\begin{array}{c}\text { Hodgkin's disease nodular sclerosis } \\
\text { stage III }\end{array}$ \\
\hline & 10020253 & $\begin{array}{c}\text { Hodgkin's disease nodular sclerosis } \\
\text { stage IV }\end{array}$ \\
\hline & 10073534 & $\begin{array}{l}\text { Hodgkin's disease nodular sclerosis } \\
\text { stage II }\end{array}$ \\
\hline & 10073535 & $\begin{array}{l}\text { Hodgkin's disease nodular sclerosis } \\
\text { stage I }\end{array}$ \\
\hline \multirow{14}{*}{ Mesothelioma } & a 10027406 & Mesothelioma \\
\hline & 10027407 & Mesothelioma malignant \\
\hline & 10027411 & Mesothelioma malignant recurrent \\
\hline & 10034480 & $\begin{array}{l}\text { Pericardial mesothelioma malignant } \\
\text { recurrent }\end{array}$ \\
\hline & 10034671 & $\begin{array}{c}\text { Peritoneal mesothelioma malignant } \\
\text { recurrent }\end{array}$ \\
\hline & 10035603 & Pleural mesothelioma \\
\hline & 10035607 & Pleural mesothelioma malignant recurrent \\
\hline & 10056558 & Peritoneal mesothelioma malignant \\
\hline & 10059518 & Pleural mesothelioma malignant \\
\hline & 10073062 & Biphasic mesothelioma \\
\hline & 10073063 & Desmoplastic mesothelioma \\
\hline & 10073064 & Epithelioid mesothelioma \\
\hline & 10073065 & Sarcomatoid mesothelioma \\
\hline & 10073066 & Pericardial mesothelioma malignant \\
\hline \multirow[t]{9}{*}{ Myeloma } & 10035222 & Plasma cell leukaemia \\
\hline & 10035226 & Plasma cell myeloma \\
\hline & 10035484 & Plasmacytoma \\
\hline & 10053869 & POEMS syndrome \\
\hline & 10060406 & Plasma cell leukaemia in remission \\
\hline & 10073132 & Plasma cell myeloma in remission \\
\hline & 10073133 & Plasma cell myeloma recurrent \\
\hline & 10078282 & Leptomeningeal myelomatosis \\
\hline & 10081847 & Plasma cell myeloma refractory \\
\hline $\begin{array}{l}\text { Merkel cell } \\
\text { carcinoma }\end{array}$ & 10029266 & Neuroendocrine carcinoma of the skin \\
\hline
\end{tabular}

HER2: Human epidermal growth factor receptor 2; POEMS: polyneuropathy, organomegaly, endocrinopathy, m-protein, and skin changes syndrome.
Table II. Preferred terms to define nephritis as an immune-related adverse event (appearing in the Medical Dictionary for Regulatory Activities version 23.1).

\begin{tabular}{|c|c|}
\hline $\begin{array}{l}\text { Preferred } \\
\text { terms number }\end{array}$ & Preferred terms \\
\hline 10018364 & Glomerulonephritis \\
\hline 10018366 & Glomerulonephritis acute \\
\hline 10018367 & Glomerulonephritis chronic \\
\hline 10018370 & Glomerulonephritis membranoproliferative \\
\hline 10018376 & Glomerulonephritis proliferative \\
\hline 10018378 & Glomerulonephritis rapidly progressive \\
\hline 10029120 & Nephritis allergic \\
\hline 10029164 & Nephrotic syndrome \\
\hline 10065673 & Nephritic syndrome \\
\hline 10066453 & Mesangioproliferative glomerulonephritis \\
\hline 10067757 & Focal segmental glomerulosclerosis \\
\hline 10073016 & Chronic autoimmune glomerulonephritis \\
\hline 10075626 & Paraneoplastic nephrotic syndrome \\
\hline 10076749 & Paraneoplastic glomerulonephritis \\
\hline 10029117 & Nephritis \\
\hline 10048302 & Tubulointerstitial nephritis \\
\hline 10069034 & Tubulointerstitial nephritis and uveitis syndrome \\
\hline 10077087 & Autoimmune nephritis \\
\hline 10083070 & Immune-mediated nephritis \\
\hline 10020586 & Hypercalcaemic nephropathy \\
\hline 10029151 & Nephropathy \\
\hline 10037111 & Pseudo-Bartter syndrome \\
\hline 10038457 & Renal glycosuria \\
\hline 10038535 & Renal tubular acidosis \\
\hline 10038536 & Renal tubular atrophy \\
\hline 10038537 & Renal tubular disorder \\
\hline 10050335 & Renal tubular dysfunction \\
\hline 10050839 & Bartter's syndrome \\
\hline 10051920 & Glomerulonephropathy \\
\hline 10052313 & Liddle's syndrome \\
\hline 10052607 & Fanconi syndrome acquired \\
\hline 10054832 & Diffuse mesangial sclerosis \\
\hline 10061989 & Glomerulosclerosis \\
\hline 10062906 & Gitelman's syndrome \\
\hline 10075849 & Potassium wasting nephropathy \\
\hline 10080593 & Pseudohypoaldosteronism \\
\hline 10083522 & Immune-mediated renal disorder \\
\hline
\end{tabular}

$(95 \% \mathrm{CI})=33.23(9.374$ to 117.8$)$ ], and pembrolizumab and lansoprazole [ROR $(95 \% \mathrm{CI})=15.63$ (3.611 to 67.69$)$ ] had a significantly higher ROR than monotherapy cases.

Multiple logistic regression analysis. In univariate analysis, the frequency of nephritis was significantly high in male patients treated with ipilimumab $(\mathrm{OR}=3.844 ; 95 \% \mathrm{CI}=1.634$ 9.042; $p=0.001)$. There were no significant differences in male patients treated with atezolizumab $(\mathrm{OR}=3.139 ; 95 \%$ $\mathrm{CI}=0.714-13.794 ; p=0.110)$, nivolumab $(\mathrm{OR}=1.371 ; 95 \%$ $\mathrm{CI}=0.913-2.060 ; p=0.126)$, and pembrolizumab $(\mathrm{OR}=1.648$; $95 \% \mathrm{CI}=0.977-2.782 ; p=0.059)$. We also conducted multiple 


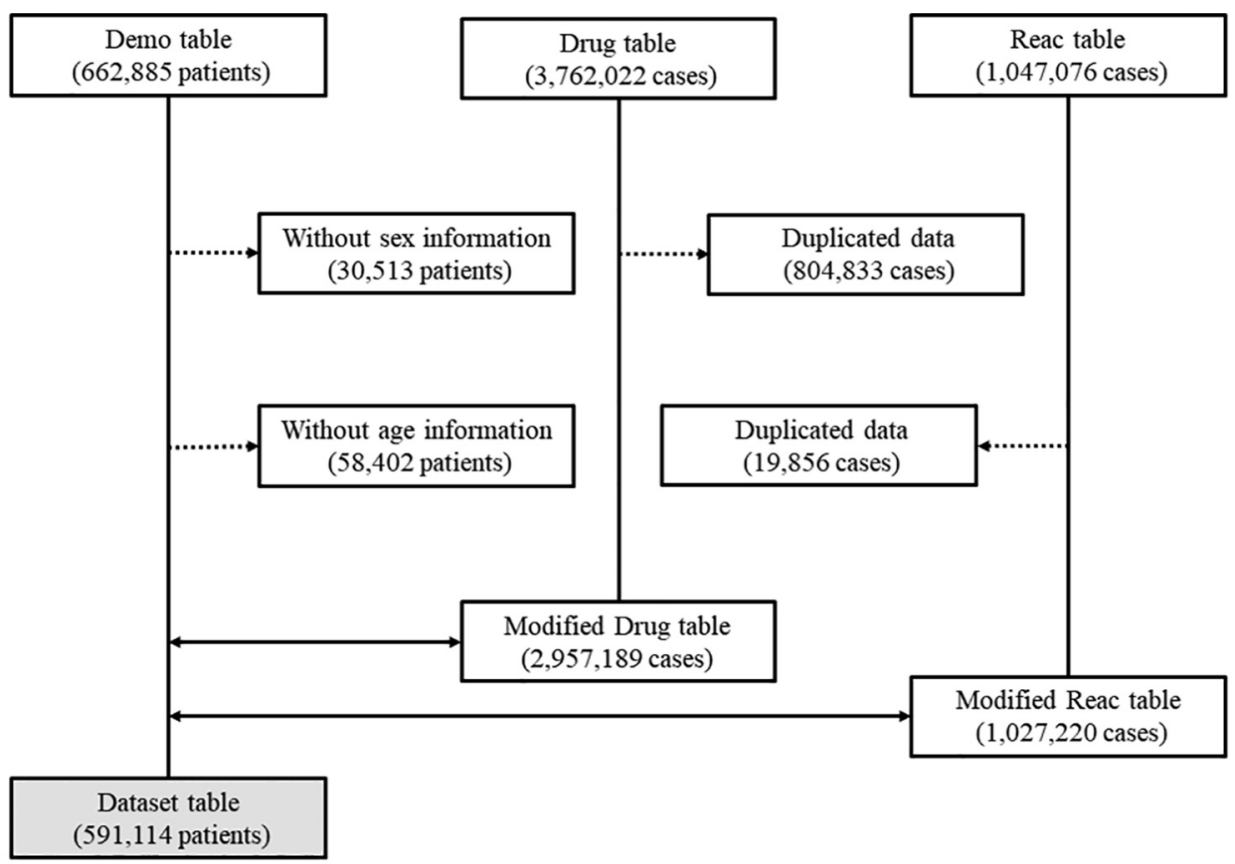

Figure 1. Flow diagram of the study. Dotted arrow and double arrow show data exclusion and combination, respectively.

Table III. Patient characteristics.

\begin{tabular}{|c|c|c|c|c|c|c|}
\hline \multirow[b]{2}{*}{ Total N } & \multirow{2}{*}{$\frac{\text { Anti PD-1 }}{\begin{array}{c}\text { Nivolumab } \\
(\mathrm{n}=9,116)\end{array}}$} & \multirow[b]{2}{*}{$\begin{array}{c}\text { Pembrolizumab } \\
(\mathrm{n}=5,838)\end{array}$} & \multirow{2}{*}{$\frac{\text { Anti CTLA-4 }}{\substack{\text { Ipilimumab } \\
(\mathrm{n}=2,831)}}$} & \multirow{2}{*}{$\frac{\text { Anti PD-L1 }}{\begin{array}{c}\text { Atezolizumab } \\
(\mathrm{n}=1,288)\end{array}}$} & \multirow[b]{2}{*}{$\begin{array}{c}\text { Durvalumab } \\
(\mathrm{n}=1,054)\end{array}$} & \multirow[b]{2}{*}{$\begin{array}{c}\text { Avelumab } \\
(\mathrm{n}=48)\end{array}$} \\
\hline & & & & & & \\
\hline Gender Male (N) & 6,626 & 4,424 & 1,883 & 911 & 849 & 27 \\
\hline Age $\geq 70$ years old $(\mathrm{N})$ & 3,990 & 3,241 & 1,142 & 624 & 550 & 32 \\
\hline \multicolumn{7}{|l|}{ With PPI use } \\
\hline Esomeprazole (N) & 17 & 15 & 7 & 3 & 1 & 0 \\
\hline Omeprazole (N) & 10 & 1 & 3 & 0 & 0 & 0 \\
\hline Vonoprazan $(\mathrm{N})$ & 12 & 13 & 3 & 10 & 1 & 0 \\
\hline Rabeprazole (N) & 13 & 5 & 2 & 3 & 0 & 0 \\
\hline Lansoprazole (N) & 37 & 19 & 11 & 4 & 3 & 0 \\
\hline
\end{tabular}

PD-1: Programmed cell death protein 1; PD-L1: programmed cell death-ligand 1; CTLA: cytotoxic T-lymphocyte-associated protein; PPI: proton pump inhibitor.

logistic regression analysis to assess the risk of ipilimumabinduced nephritis. The frequency of nephritis was significantly higher in male patients treated with ipilimumab $(\mathrm{OR}=3.798 ; 95 \% \mathrm{CI}=1.614-8.938 ; p=0.002)$. Age over 70 years did not influence the frequency of nephritis (Table V).

\section{Discussion}

The estimated incidence of ICPI-induced nephritis is much lower than that of other irAEs (26). Therefore, understanding ICPI-induced nephritis was limited to small case series. To clearly identify the risk factors for ICPI-induced nephritis, Cortazar et al. (13) conducted a multicenter study involving 138 patients with ICPI-induced nephritis. This report identified low baseline eGFR and PPI use as independent risk factors of ICPIinduced nephritis. However, the PPIs that increase the risk of ICPI-induced nephritis were not identified in this study. In the present study, omeprazole increased the frequency of nephritis in patients treated with ipilimumab or nivolumab. Esomeprazole and lansomeprazole increased the frequency of nivolumab and pembrolizumab-induced nephritis. Furthermore, the frequency of ipilimumab-induced nephritis increased in male patients. 
Table IV. Crude reporting odds ratios for nephritis.

\begin{tabular}{|c|c|c|c|c|}
\hline Concomitant drug & Drug & Case of nephritis & Total SE & ROR $(95 \% \mathrm{CI})$ \\
\hline \multirow{11}{*}{ Monotherapy } & Atezolizumab & 17 & 1,288 & $1.780(1.102-2.874)$ \\
\hline & Avelumab & 0 & 48 & N.A \\
\hline & Ipilimumab & 51 & 2,831 & $2.454(1.857-3.242)$ \\
\hline & Durvalumab & 2 & 1,054 & $0.252(0.063-1.010)$ \\
\hline & Nivolumab & 139 & 9,116 & $2.091(1.764-2.479)$ \\
\hline & Pembrolizumab & 104 & 5,838 & $2.443(2.008-2.973)$ \\
\hline & Esomeprazole & 20 & 1,310 & $2.064(1.326-3.214)$ \\
\hline & Omeprazole & 51 & 1,660 & $4.248(3.209-5.622)$ \\
\hline & Vonoprazan & 17 & 1,254 & $1.829(1.132-2.954)$ \\
\hline & Rabeprazole & 35 & 1,510 & $3.169(2.263-4.437)$ \\
\hline & Lansoprazole & 66 & 4,123 & $2.178(1.705-2.783)$ \\
\hline \multirow[t]{5}{*}{ Atezolizumab } & Esomeprazole & 0 & 3 & N.A \\
\hline & Omeprazole & 0 & 0 & N.A \\
\hline & Vonoprazan & 1 & 10 & $14.76(1.870-116.5)$ \\
\hline & Rabeprazole & 1 & 3 & $66.43(6.022-732.8)$ \\
\hline & Lansoprazole & 0 & 4 & N.A \\
\hline \multirow[t]{5}{*}{ Avelumab } & Esomeprazole & 0 & 0 & N.A \\
\hline & Omeprazole & 0 & 0 & N.A \\
\hline & Vonoprazan & 0 & 0 & N.A \\
\hline & Rabeprazole & 0 & 0 & N.A \\
\hline & Lansoprazole & 0 & 0 & N.A \\
\hline \multirow[t]{5}{*}{ Ipilimumab } & Esomeprazole & 0 & 7 & 0 \\
\hline & Omeprazole & 2 & 3 & $265.8(24.09-2931)$ \\
\hline & Vonoprazan & 0 & 3 & 0 \\
\hline & Rabeprazole & 0 & 2 & 0 \\
\hline & Lansoprazole & 2 & 11 & $29.53(6.378-136.7)$ \\
\hline \multirow[t]{5}{*}{ Durvalumab } & Esomeprazole & 0 & 1 & N.A \\
\hline & Omeprazole & 0 & 0 & N.A \\
\hline & Vonoprazan & 0 & 1 & N.A \\
\hline & Rabeprazole & 0 & 0 & N.A \\
\hline & Lansoprazole & 0 & 3 & N.A \\
\hline \multirow[t]{5}{*}{ Nivolumab } & Esomeprazole & 4 & 17 & $40.91(13.33-125.5)$ \\
\hline & Omeprazole & 6 & 10 & $199.5(56.28-707.3)$ \\
\hline & Vonoprazan & 0 & 12 & N.A \\
\hline & Rabeprazole & 6 & 13 & $114.0(38.30-339.4)$ \\
\hline & Lansoprazole & 7 & 37 & $31.04(13.67-70.71)$ \\
\hline \multirow[t]{5}{*}{ Pembrolizumab } & Esomeprazole & 3 & 15 & $33.23(9.374-117.8)$ \\
\hline & Omeprazole & 0 & 1 & N.A \\
\hline & Vonoprazan & 0 & 13 & N.A \\
\hline & Rabeprazole & 1 & 5 & $33.21(3.711-297.2)$ \\
\hline & Lansoprazole & 2 & 19 & $15.63(3.611-67.69)$ \\
\hline
\end{tabular}

SE: Side effect; ROR: reporting odds ratio; CI: confidence interval; N.A: not available.

Hypomagnesemia, AKI, AIN, and CKD were reported as adverse events associated with PPI use $(30,31)$. The risk of hospital admission was elevated within 120 days of PPI exposure (32). In addition, PPI use was associated with increasing mortality due to cardiovascular disease, CKD, and upper gastrointestinal cancer (33). These previous reports suggested that medical practitioners should consider the potential benefits and risks of PPIs. Based on kidney biopsy results, the frequency of AIN with severe inflammatory cell infiltration was higher in ICPI-induced nephritis than in other types of renal injury (14). Hence, these reports suggest that cell-mediated immunity is associated with nephritis. Although the underlying mechanism of PPI-induced nephritis is unclear, PPI-induced nephritis showed cellular infiltrates with lymphocytes and occasional eosinophils in the renal interstitium $(20,22)$. Therefore, concomitant use of ICPI and PPI might develop cell-mediated immunity associated with AIN. In the present study, omeprazole and lansoprazole showed high risk of nephritis induction in the cases with or without ICPI. Although the most of omeprazole-induced nephritis are recognized as interstitial damage $(17,30)$, the underlying mechanism is unclear. 
Table V. Univariate and multivariate analysis for predictors of ICPI-induced nephritis.

\begin{tabular}{|c|c|c|c|c|}
\hline & \multicolumn{2}{|c|}{ Univariate analysis } & \multicolumn{2}{|c|}{ Multivariate analysis } \\
\hline & OR $(95 \% \mathrm{CI})$ & $p$-Value & OR $(95 \% \mathrm{CI})$ & $p$-Value \\
\hline \multicolumn{5}{|l|}{ Atezolizumab } \\
\hline Male & $3.139(0.714-13.79)$ & 0.110 & & \\
\hline$\geq 70$ years & $1.200(0.460-3.130)$ & 0.709 & & \\
\hline \multicolumn{5}{|l|}{ Ipilimumab } \\
\hline Male & $3.844(1.634-9.042)$ & 0.001 & $3.798(1.614-8.938)$ & 0.002 \\
\hline$\geq 70$ years & $1.432(0.822-2.492)$ & 0.202 & $1.386(0.795-2.415)$ & 0.250 \\
\hline \multicolumn{5}{|l|}{ Nivolumab } \\
\hline Male & $1.371(0.913-2.060)$ & 0.126 & & \\
\hline$\geq 70$ years & $0.975(0.695-1.368)$ & 0.885 & & \\
\hline \multicolumn{5}{|c|}{ Pembrolizumab } \\
\hline Male & $1.648(0.977-2.782)$ & 0.059 & & \\
\hline$\geq 70$ years & $0.934(0.633-1.378)$ & 0.730 & & \\
\hline
\end{tabular}

ICPI: Immune checkpoint inhibitor; OR: odds ratio; CI: confidence interval.

Organic cation transporters (OCTs) uptake PPIs to renal tubular cells (34). Since the affinity for OCTs and accumulation in renal tubular cells are higher for omeprazole or lansoprazole than that for rabeprazole (34), omeprazole, and lansoprazole have more potential in inducing AIN compared to other PPIs.

Male gender showed an increasing tendency towards risk of ICPI-induced nephritis (13). Although the mechanism of nephritis in male patients treated with ICPIs was unclear, our results supported this previous report. Since the frequency of irAEs is higher in female patients than that in male patients (35-37), ICPI-induced nephritis might have different mechanism to that of other irAEs. Since ipilimumab has an immunoglobulin $\mathrm{G}_{1}\left(\mathrm{IgG}_{1}\right)$ structure, it might lead to higher activation of complement and other immune system factors than the rest of the $\operatorname{IgG}$ subtypes (38-40). Therefore, our results suggested that male patients or patients with concomitant use of ICPIs and PPIs (excluded vonoprazan) should be monitored for renal function after chemotherapy.

The present study has certain limitations. First, the ADE signal of avelumab-induced nephritis was either weak or not detected because of the small sample size. Nivolumab was approved in Japan in 2014, whereas ipilimumab, pembrolizumab, avelumab, atezolizumab, and durvalumab were approved in 2016, 2015, 2017, 2018, and 2018, respectively. Therefore, the number of ADE reports for nivolumab is greater than those for the other ICPIs. Second, as a large spontaneous reporting system, the JADER database has various biases including under- or over-reporting and confounders caused by comorbidities $(28,41-45)$. Third, the number of nephritis event was small in concomitant use of ICPI and PPI. Although multiple logistic regression analysis could be conducted in monotherapy data set, this analysis was not applied for concomitant use data set because of lack statistical power.
The most common trigger of AIN is the drug used (46), therefore the identification of the types of drug is important in determining a preventive strategy. Although our results provide new insights of ICPI- and PPI-induced nephritis, further basic and clinical studies are required to elucidate the mechanisms of action.

\section{Conflicts of Interest}

The Authors report no conflicts of interest regarding this work.

\section{Authors' Contributions}

KK, TM, YI, and NT designed this study. KK and TK carried out the survey of the JADER database. KK, TM, TK, and $\mathrm{MH}$ performed the statistical analyses. KK, TM, YI, KT, SY, and NT drafted the manuscript. All Authors approved the final manuscript.

\section{Acknowledgements}

The Authors would like to thank Editage (https://www.editage.com/) for editing and reviewing this manuscript for English language.

\section{References}

1 Motzer RJ, Escudier B, McDermott DF, George S, Hammers HJ, Srinivas S, Tykodi SS, Sosman JA, Procopio G, Plimack ER, Castellano D, Choueiri TK, Gurney H, Donskov F, Bono P, Wagstaff J, Gauler TC, Ueda T, Tomita Y, Schutz FA, Kollmannsberger C, Larkin J, Ravaud A, Simon JS, Xu LA, Waxman IM, Sharma P and CheckMate 025 Investigators: Nivolumab versus everolimus in advanced renal-cell carcinoma. N Engl J Med 373(19): 1803-1813, 2015. PMID: 26406148. DOI: 10.1056/NEJMoa1510665

2 Borghaei H, Paz-Ares L, Horn L, Spigel DR, Steins M, Ready NE, Chow LQ, Vokes EE, Felip E, Holgado E, Barlesi F, 
Kohlhäufl M, Arrieta O, Burgio MA, Fayette J, Lena H, Poddubskaya E, Gerber DE, Gettinger SN, Rudin CM, Rizvi N, Crinò L, Blumenschein GR Jr, Antonia SJ, Dorange C, Harbison $\mathrm{CT}$, Graf Finckenstein $\mathrm{F}$ and Brahmer JR: Nivolumab versus docetaxel in advanced nonsquamous non-small-cell lung cancer. N Engl J Med 373(17): 1627-1639, 2015. PMID: 26412456. DOI: $10.1056 /$ NEJMoa1507643

3 Brahmer JR, Tykodi SS, Chow LQ, Hwu WJ, Topalian SL, Hwu P, Drake CG, Camacho LH, Kauh J, Odunsi K, Pitot HC, Hamid O, Bhatia S, Martins R, Eaton K, Chen S, Salay TM, Alaparthy S, Grosso JF, Korman AJ, Parker SM, Agrawal S, Goldberg SM, Pardoll DM, Gupta A and Wigginton JM: Safety and activity of anti-PD-L1 antibody in patients with advanced cancer. N Engl J Med 366(26): 2455-2465, 2012. PMID: 22658128. DOI: 10.1056/NEJMoa1200694

4 Hodi FS, O’Day SJ, McDermott DF, Weber RW, Sosman JA, Haanen JB, Gonzalez R, Robert C, Schadendorf D, Hassel JC, Akerley W, van den Eertwegh AJ, Lutzky J, Lorigan P, Vaubel JM, Linette GP, Hogg D, Ottensmeier CH, Lebbé C, Peschel C, Quirt I, Clark JI, Wolchok JD, Weber JS, Tian J, Yellin MJ, Nichol GM, Hoos A and Urba WJ: Improved survival with ipilimumab in patients with metastatic melanoma. N Engl J Med 363(8): 711723, 2010. PMID: 20525992. DOI: 10.1056/NEJMoa1003466

5 Robert C, Schachter J, Long GV, Arance A, Grob JJ, Mortier L, Daud A, Carlino MS, McNeil C, Lotem M, Larkin J, Lorigan P, Neyns B, Blank CU, Hamid O, Mateus C, Shapira-Frommer R, Kosh M, Zhou H, Ibrahim N, Ebbinghaus S, Ribas A and KEYNOTE-006 investigators: Pembrolizumab versus ipilimumab in advanced melanoma. N Engl J Med 372(26): 2521-2532, 2015. PMID: 25891173. DOI: 10.1056/NEJMoa1503093

6 Brahmer JR, Drake CG, Wollner I, Powderly JD, Picus J, Sharfman WH, Stankevich E, Pons A, Salay TM, McMiller TL, Gilson MM, Wang C, Selby M, Taube JM, Anders R, Chen L, Korman AJ, Pardoll DM, Lowy I and Topalian SL: Phase I study of single-agent anti-programmed death-1 (MDX-1106) in refractory solid tumors: safety, clinical activity, pharmacodynamics, and immunologic correlates. J Clin Oncol 28(19): 3167-3175, 2010. PMID: 20516446. DOI: 10.1200/JCO.2009.26.7609

7 Herbst RS, Soria JC, Kowanetz M, Fine GD, Hamid O, Gordon MS, Sosman JA, McDermott DF, Powderly JD, Gettinger SN, Kohrt HE, Horn L, Lawrence DP, Rost S, Leabman M, Xiao Y, Mokatrin A, Koeppen H, Hegde PS, Mellman I, Chen DS and Hodi FS: Predictive correlates of response to the anti-PD-L1 antibody MPDL3280A in cancer patients. Nature 515(7528): 563-567, 2014. PMID: 25428504. DOI: 10.1038/nature 14011

8 Melero I, Hervas-Stubbs S, Glennie M, Pardoll DM and Chen L: Immunostimulatory monoclonal antibodies for cancer therapy. Nat Rev Cancer 7(2): 95-106, 2007. PMID: 17251916. DOI: $10.1038 / \mathrm{nrc} 2051$

9 Tie Y, Ma X, Zhu C, Mao Y, Shen K, Wei X, Chen Y and Zheng $\mathrm{H}$ : Safety and efficacy of nivolumab in the treatment of cancers: A meta-analysis of 27 prospective clinical trials. Int J Cancer 140(4): 948-958, 2017. PMID: 27813059. DOI: 10.1002/ ijc. 30501

10 Bashey A, Medina B, Corringham S, Pasek M, Carrier E, Vrooman L, Lowy I, Solomon SR, Morris LE, Holland HK, Mason JR, Alyea EP, Soiffer RJ and Ball ED: CTLA4 blockade with ipilimumab to treat relapse of malignancy after allogeneic hematopoietic cell transplantation. Blood 113(7): 1581-1588, 2009. PMID: 18974373. DOI: 10.1182/blood-2008-07-168468
11 Sukari A, Nagasaka M, Alhasan R, Patel D, Wozniak A, Ramchandren R, Vaishampayan U, Weise A, Flaherty L, Jang H, Kim S and Gadgeel S: Cancer site and adverse events induced by immune checkpoint inhibitors: A retrospective analysis of reallife experience at a single institution. Anticancer Res 39(2): 781790, 2019. PMID: 30711957. DOI: 10.21873/anticanres.13175

12 Fadel F, El Karoui K and Knebelmann B: Anti-CTLA4 antibodyinduced lupus nephritis. N Engl J Med 361(2): 211-212, 2009. PMID: 19587352. DOI: 10.1056/NEJMc0904283

13 Cortazar FB, Kibbelaar ZA, Glezerman IG, Abudayyeh A, Mamlouk O, Motwani SS, Murakami N, Herrmann SM, Manohar S, Shirali AC, Kitchlu A, Shirazian S, Assal A, Vijayan A, Renaghan AD, Ortiz-Melo DI, Rangarajan S, Malik AB, Hogan JJ, Dinh AR, Shin DS, Marrone KA, Mithani Z, Johnson DB, Hosseini A, Uprety D, Sharma S, Gupta S, Reynolds KL, Sise ME and Leaf DE: Clinical features and outcomes of immune checkpoint inhibitor-associated AKI: A multicenter study. J Am Soc Nephrol 31(2): 435-446, 2020. PMID: 31896554. DOI: 10.1681/ASN.2019070676

14 Izzedine H, Mateus C, Boutros C, Robert C, Rouvier P, Amoura $\mathrm{Z}$ and Mathian A: Renal effects of immune checkpoint inhibitors. Nephrol Dial Transplant 32(6): 936-942, 2017. PMID: 28025384. DOI: $10.1093 /$ ndt/gfw382

15 Espi M, Teuma C, Novel-Catin E, Maillet D, Souquet PJ, Dalle S, Koppe L and Fouque D: Renal adverse effects of immune checkpoints inhibitors in clinical practice: ImmuNoTox study. Eur J Cancer 147: 29-39, 2021. PMID: 33607383. DOI: 10.1016/j.ejca.2021.01.005

16 Meraz-Muñoz A, Amir E, Ng P, Avila-Casado C, Ragobar C, Chan C, Kim J, Wald R and Kitchlu A: Acute kidney injury associated with immune checkpoint inhibitor therapy: incidence, risk factors and outcomes. J Immunother Cancer 8(1): e000467, 2020. PMID: 32601079. DOI: 10.1136/jitc-2019-000467

17 d'Adamo G, Spinelli C, Forte F and Gangeri F: Omeprazoleinduced acute interstitial nephritis. Ren Fail 19(1): 171-175, 1997. PMID: 9044464 . DOI: 10.3109/08860229709026272

18 Torpey N, Barker T and Ross C: Drug-induced tubulo-interstitial nephritis secondary to proton pump inhibitors: experience from a single UK renal unit. Nephrol Dial Transplant 19(6): 14411446, 2004. PMID: 15004262. DOI: 10.1093/ndt/gfh137

19 Muriithi AK, Leung N, Valeri AM, Cornell LD, Sethi S, Fidler ME and Nasr SH: Clinical characteristics, causes and outcomes of acute interstitial nephritis in the elderly. Kidney Int 87(2): 458-464, 2015. PMID: 25185078. DOI: 10.1038/ki.2014.294

20 Geevasinga N, Coleman PL and Roger SD: Rabeprazole-induced acute interstitial nephritis. Nephrology (Carlton) 10(1): 7-9, 2005. PMID: 15705174. DOI: 10.1111/j.1440-1797.2005.00365.x

21 Jones B, Hewson E and Price A: Acute interstitial nephritis due to omeprazole. Lancet 344(8928): 1017-1018, 1994. PMID: 7934394. DOI: 10.1016/s0140-6736(94)91674-8

$22 \mathrm{Ni} \mathrm{N}$, Moeckel GW and Kumar C: Late-onset omeprazoleassociated acute interstitial nephritis. J Am Geriatr Soc 58(12): 2443-2444, 2010. PMID: 21143456. DOI: 10.1111/j.15325415.2010.03194.x

23 Lazarus B, Chen Y, Wilson FP, Sang Y, Chang AR, Coresh J and Grams ME: Proton Pump Inhibitor Use and the Risk of Chronic Kidney Disease. JAMA Intern Med 176(2): 238-246, 2016. PMID: 26752337. DOI: 10.1001/jamainternmed.2015.7193

24 Xie Y, Bowe B, Li T, Xian H, Balasubramanian S and Al-Aly Z: Proton pump inhibitors and risk of incident CKD and 
progression to ESRD. J Am Soc Nephrol 27(10): 3153-3163, 2016. PMID: 27080976. DOI: 10.1681/ASN.2015121377

25 Nochaiwong S, Ruengorn C, Awiphan R, Koyratkoson K, Chaisai C, Noppakun K, Chongruksut W and Thavorn K: The association between proton pump inhibitor use and the risk of adverse kidney outcomes: a systematic review and metaanalysis. Nephrol Dial Transplant 33(2): 331-342, 2018. PMID: 28339835. DOI: $10.1093 / \mathrm{ndt} / \mathrm{gfw} 470$

26 Cortazar FB, Marrone KA, Troxell ML, Ralto KM, Hoenig MP, Brahmer JR, Le DT, Lipson EJ, Glezerman IG, Wolchok J, Cornell LD, Feldman P, Stokes MB, Zapata SA, Hodi FS, Ott PA, Yamashita M and Leaf DE: Clinicopathological features of acute kidney injury associated with immune checkpoint inhibitors. Kidney Int 90(3): 638-647, 2016. PMID: 27282937. DOI: 10.1016/j.kint.2016.04.008

27 Hasegawa S, Ikesue H, Nakao S, Shimada K, Mukai R, Tanaka M, Matsumoto K, Inoue M, Satake R, Yoshida Y, Goto F, Hashida T and Nakamura M: Analysis of immune-related adverse events caused by immune checkpoint inhibitors using the Japanese Adverse Drug Event Report database. Pharmacoepidemiol Drug Saf 29(10): 1279-1294, 2020. PMID: 32869941. DOI: 10.1002/pds.5108

28 Sugawara H, Uchida M, Suzuki S, Suga Y, Uesawa Y, Nakagawa $\mathrm{T}$ and Takase $\mathrm{H}$ : Analyses of respiratory depression associated with opioids in cancer patients based on the Japanese adverse drug event report database. Biol Pharm Bull 42(7): 1185-1191, 2019. PMID: 31257293. DOI: 10.1248/bpb.b19-00105

29 Li H, Deng J, Yue Z, Zhang Y and Sun H: Detecting drug-herbal interaction using a spontaneous reporting system database: an example with benzylpenicillin and qingkailing injection. Eur J Clin Pharmacol 71(9): 1139-1145, 2015. PMID: 26159784. DOI: 10.1007/s00228-015-1898-8

30 Al-Aly Z, Maddukuri G and Xie Y: Proton pump inhibitors and the kidney: Implications of current evidence for clinical practice and when and how to deprescribe. Am J Kidney Dis 75(4): $497-$ 507, 2020. PMID: 31606235. DOI: 10.1053/j.ajkd.2019.07.012

31 Hart E, Dunn TE, Feuerstein S and Jacobs DM: Proton pump inhibitors and risk of acute and chronic kidney disease: A retrospective cohort study. Pharmacotherapy 39(4): 443-453, 2019. PMID: 30779194. DOI: 10.1002/phar.2235

32 Antoniou T, Macdonald EM, Hollands S, Gomes T, Mamdani MM, Garg AX, Paterson JM and Juurlink DN: Proton pump inhibitors and the risk of acute kidney injury in older patients: a population-based cohort study. CMAJ Open 3(2): E166-E171, 2015. PMID: 26389094. DOI: 10.9778/cmajo.20140074

33 Xie Y, Bowe B, Yan Y, Xian H, Li T and Al-Aly Z: Estimates of all cause mortality and cause specific mortality associated with proton pump inhibitors among US veterans: cohort study. BMJ 365: 11580, 2019. PMID: 31147311. DOI: 10.1136/bmj.11580

34 Nies AT, Hofmann U, Resch C, Schaeffeler E, Rius M and Schwab M: Proton pump inhibitors inhibit metformin uptake by organic cation transporters (OCTs). PLoS One 6(7): e22163, 2011. PMID: 21779389. DOI: 10.1371/journal.pone.0022163

35 Zamami Y, Niimura T, Okada N, Koyama T, Fukushima K, IzawaIshizawa $\mathrm{Y}$ and Ishizawa $\mathrm{K}$ : Factors associated with immune checkpoint inhibitor-related myocarditis. JAMA Oncol 5(11): 16351637, 2019. PMID: 31436802. DOI: 10.1001/jamaoncol.2019.3113

36 Suresh K, Voong KR, Shankar B, Forde PM, Ettinger DS, Marrone KA, Kelly RJ, Hann CL, Levy B, Feliciano JL, Brahmer JR, Feller-Kopman D, Lerner AD, Lee H, Yarmus L, D'Alessio F, Hales RK, Lin CT, Psoter KJ, Danoff SK and
Naidoo J: Pneumonitis in non-small cell lung cancer patients receiving immune checkpoint immunotherapy: Incidence and risk factors. J Thorac Oncol 13(12): 1930-1939, 2018. PMID: 30267842. DOI: $10.1016 /$ j.jtho.2018.08.2035

37 Duma N, Abdel-Ghani A, Yadav S, Hoversten KP, Reed CT, Sitek AN, Enninga EAL, Paludo J, Aguilera JV, Leventakos K, Lou Y, Kottschade LA, Dong H, Mansfield AS, Manochakian R, Adjei AA and Dronca RS: Sex differences in tolerability to antiprogrammed cell death protein 1 therapy in patients with metastatic melanoma and non-small cell lung cancer: Are we all equal? Oncologist 24(11): e1148-e1155, 2019. PMID: 31036771. DOI: $10.1634 /$ theoncologist.2019-0094

38 Deveuve Q, Lajoie L, Barrault B and Thibault G: The proteolytic cleavage of therapeutic monoclonal antibody hinge region: More than a matter of subclass. Front Immunol 11: 168, 2020. PMID: 32117299. DOI: 10.3389/fimmu.2020.00168

39 Sondermann P, Huber R, Oosthuizen V and Jacob U: The 3.2-A crystal structure of the human IgG1 Fc fragment-Fc gammaRIII complex. Nature 406(6793): 267-273, 2000. PMID: 10917521. DOI: $10.1038 / 35018508$

40 Ugurlar D, Howes SC, de Kreuk BJ, Koning RI, de Jong RN, Beurskens FJ, Schuurman J, Koster AJ, Sharp TH, Parren PWHI and Gros P: Structures of C1-IgG1 provide insights into how danger pattern recognition activates complement. Science 359(6377): 794-797, 2018. PMID: 29449492. DOI: 10.1126/ science.aao4988

41 Abe J, Umetsu R, Mataki K, Kato Y, Ueda N, Nakayama Y, Hane Y, Matsui T, Hatahira H, Sasaoka S, Motooka Y, Hara H, Kato Z, Kinosada Y, Inagaki N and Nakamura M: Analysis of Stevens-Johnson syndrome and toxic epidermal necrolysis using the Japanese Adverse Drug Event Report database. J Pharm Health Care Sci 2: 14, 2016. PMID: 27330825. DOI: 10.1186/ s40780-016-0048-5

42 Hosoya R, Uesawa Y, Ishii-Nozawa R and Kagaya H: Analysis of factors associated with hiccups based on the Japanese Adverse Drug Event Report database. PLoS One 12(2): e0172057, 2017. PMID: 28196104. DOI: 10.1371/journal.pone.0172057

43 Hara A, Matsumoto K, Yokoyama Y and Kizu J: Factorial analysis of hepatitis $\mathrm{B}$ virus reactivation-induced hepatitis $\mathrm{B}$ using JADER. Biol Pharm Bull 40(6): 782-788, 2017. PMID: 28566622. DOI: 10.1248/bpb.b16-00765

44 Hasegawa S, Matsui T, Hane Y, Abe J, Hatahira H, Motooka Y, Sasaoka S, Fukuda A, Naganuma M, Hirade K, Takahashi Y, Kinosada Y and Nakamura M: Thromboembolic adverse event study of combined estrogen-progestin preparations using Japanese Adverse Drug Event Report database. PLoS One 12(7): e0182045, 2017. PMID: 28732067. DOI: 10.1371/journal.pone.0182045

45 Takada S, Hirokazu H, Yamagishi K, Hideki S and Masayuki E: Predictors of the onset of type 1 diabetes obtained from realworld data analysis in cancer patients treated with immune checkpoint inhibitors. Asian Pac J Cancer Prev 21(6): 1697-1699, 2020. PMID: 32592366. DOI: 10.31557/APJCP.2020.21.6.1697

46 Regusci A, Lava SAG, Milani GP, Bianchetti MG, Simonetti GD and Vanoni F: Tubulointerstitial nephritis and uveitis syndrome: a systematic review. Nephrol Dial Transplant, 2021. PMID: 33561271. DOI: $10.1093 /$ ndt/gfab030

Received May 13, 2021

Revised June 7, 2021

Accepted June 14, 2021 